\title{
The Surface of Stellar Models - Now with more 3D simulations!
}

Regner Trampedach ${ }^{1,2, a}$, Jørgen Christensen-Dalsgaard ${ }^{1}$, Martin Asplund ${ }^{3}$, Robert F. Stein ${ }^{4}$, and Åke Nordlund $^{5}$

1 Stellar Astrophysics Centre, Department of Physics and Astronomy, Aarhus University, DK-8000 Aarhus C, Denmark

2 JILA, University of Colorado and National Institute of Standards and Technology, 440 UCB, Boulder, CO 80309, USA

3 Research School of Astronomy and Astrophysics, Mt. Stromlo Observatory, Cotter Road, Weston ACT 2611, Australia

4 Astronomical Observatory/Niels Bohr Institute, Juliane Maries Vej 30, DK-2100 Copenhagen Ø, Denmark

\begin{abstract}
We have constructed a grid of 3D hydrodynamic simulations of deep convective and line-blanketed atmospheres. We have developed a new consistent method for computing and employing $T(\tau)$ relations from these simulations, as surface boundary conditions for 1D stellar structure models. These 1D models have, in turn, had their mixinglength, $\alpha$, calibrated against the averaged structure of each of the simulations. Both $\alpha$ and $T(\tau)$ vary significantly with $T_{\text {eff }}$ and $\log g$.
\end{abstract}

\section{Introduction}

A major uncertainty of current modelling of stars, is the treatment of convection. Being a dynamic, non-linear and inherently 3D phenomenon, simple prescriptions to be used in 1D stellar structure models are hard to come by. This is the main reason that the formulation in most widespread use, the mixing-length theory (MLT) [1], has survived for more than half a century. It does not describe the surface layers well, but below the photosphere and the super-adiabatic peak, the structure of convective envelopes is well described by MLT [5]. MLT suffers from free parameters, with little guidance from theory as to their values. First principles, hydrodynamical simulations of convection is the most promising means for learning about convection, and our first step has been to calibrate the main free parameter, $\alpha$, of MLT, scaling the mixing-length, $\Lambda=\alpha H_{p}$, in terms of the local pressure scale-height, $H_{p}$.

\section{Results}

We used the grid of solar metallicity convection simulations, as described in detail by [2]. The first step in the $\alpha$ calibration, was to develop an outer boundary condition for the 1D structure models, that is consistent with the 3D simulations. This was carried out through the calculation of $T(\tau)$ relations between temperature and Rosseland optical depth, $\tau$, presented in [3]. That paper also describes a new formulation for evaluating $T(\tau)$ relations from atmosphere models (1D or 3D, that solve the radiative transfer), and their subsequent implementation in 1D structure models. The result is a formulation that is physically consistent despite possible differences between the atmosphere and interior models. This formulation also applies throughout the structure model eliminating the need for a matching point between interior and atmospheric formulations in the stellar structure model.

\footnotetext{
a e-mail: trampeda@lcd.colorado.edu
}

This is an Open Access article distributed under the terms of the Creative Commons Attribution License 4.0, which permits unrestricted use, distribution, and reproduction in any medium, provided the original work is properly cited. 


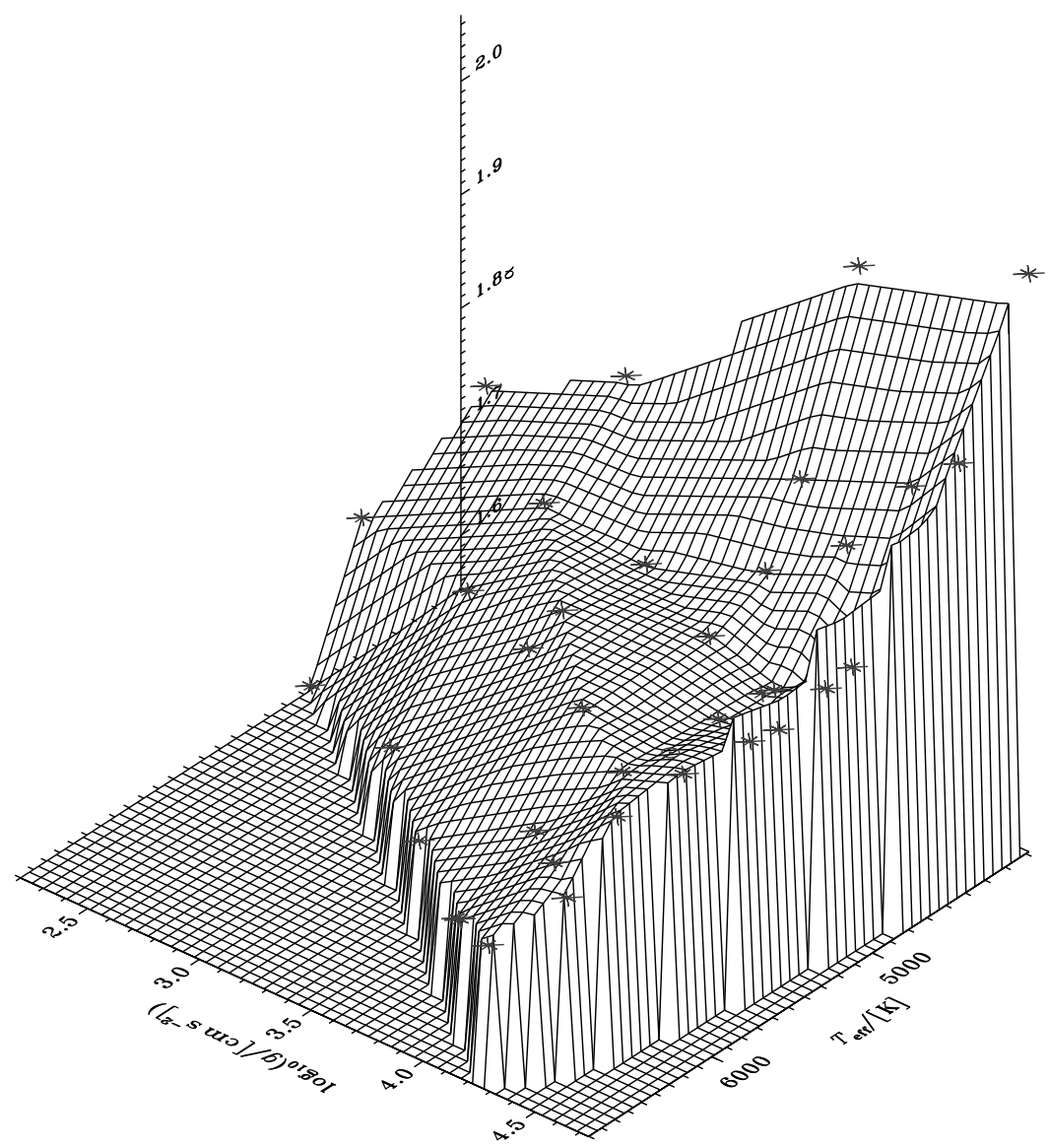

Fig. 1. The mixing-length, $\alpha$, as calibrated against our grid of 3D convection simulations, and as function of $T_{\text {eff }}$ and $\log g$. The front of the plot shows the main sequence, from $4185 \mathrm{~K}$ to $6902 \mathrm{~K}$, with $\alpha$-values of 2.06 and 1.68 , respectively.

The $\alpha$ calibration consists of matching density and temperature of the 1D model with those of the averaged 3D simulation, at a common pressure point. This calibration is detailed in [4], and shown in Fig. 1. There it was also found that the $T(\tau)$ relations have a significant impact on the calibration, both in terms of the resulting $\alpha$ values and the resulting depth of the convective envelopes. The atmopsheric opacity is the third component of the $\alpha$ calibration, and we have used the exact same opacities in the 1D models as in the 3D simulations. Details about accessing the data for implementing these results, are given in [4].

\section{References}

1. Böhm-Vitense E., Zs. f. Astroph., 46, 108 (1958)

2. Trampedach R., Asplund M., Collet R., Nordlund Å., Stein R. F., ApJ 769, 18 (2013)

3. Trampedach R., Christensen-Dalsgaard J., Nordlund A., Asplund M., Stein R. F., MNRAS 442, 805 (2014)

4. Trampedach R., Christensen-Dalsgaard J., Nordlund Å, Asplund M., Stein R. F., MNRAS 445, 4366 (2014)

5. Trampedach R., Stein R. F., ApJ 731, 78 (2011) 